Canadian

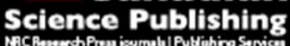

Applied Physiology, Nutrition, and Metabolism Physiologie appliquée, nutrition et métabolisme

\title{
EFFECTS OF ISCHEMIC PRECONDITIONING ON SHORT- DURATION CYCLING PERFORMANCE
}

\begin{tabular}{|r|l|}
\hline Journal: & Applied Physiology, Nutrition, and Metabolism \\
\hline Manuscript ID & apnm-2015-0646.R1 \\
\hline Manuscript Type: & Article \\
\hline Complete List of Authors: & $\begin{array}{l}\text { Cruz, Rogério; Santa Catarina State University, Center for Health and } \\
\text { Sports Sciences } \\
\text { Aguiar, Rafael; Santa Catarina State University, Center for Health and } \\
\text { Sports Sciences } \\
\text { Turnes, Tiago; Santa Catarina State University, Center for Health and } \\
\text { Sport Sciences } \\
\text { Salvador, Amadeo; Santa Catarina State University, Center for Health and } \\
\text { Sport Sciences } \\
\text { Caputo, Fabrizio; Santa Catarina State University, Center for Health and } \\
\text { Sport Sciences }\end{array}$ \\
\hline Keyword: & $\begin{array}{l}\text { accumulated oxygen deficit, anaerobic exercise, blood lactate kinetics, } \\
\text { EPOC, ergogenic aid }\end{array}$ \\
\hline
\end{tabular}




\section{EFFECTS OF ISCHEMIC PRECONDITIONING ON SHORT-DURATION CYCLING PERFORMANCE}

Rogério Santos de Oliveira $\mathrm{Cruz}^{1}$

Rafael Alves de Aguiar ${ }^{1}$

Tiago Turnes ${ }^{1}$

Amadeo Félix Salvador ${ }^{1}$

Fabrizio Caputo ${ }^{1}$

1 - Human Performance Research Group, College of Health and Sport Science, Santa Catarina State University, Florianópolis, Brazil.

IPC AND SHORT-DURATION CYCLING PERFORMANCE

R. Cruz ( $\square)$

Laboratório de Pesquisas em Desempenho Humano - CEFID/UDESC

Rua Pascoal Simone, 358, Coqueiros - Florianópolis - SC - Brasil - CEP - 88080-350.

Phone: +55483321.8641

Fax: +55483321.8607

E-mail: cruz.rso@gmail.com 


\section{ABSTRACT}

Purpose: It has been demonstrated that ischemic preconditioning (IPC) improves endurance performance. However, the potential benefits during anaerobic events and the mechanism(s) underlying these benefits remain unclear. Methods: Fifteen recreational cyclists were assessed in order to evaluate the effects of IPC of the upper thighs on anaerobic performance, skeletal muscle activation and metabolic responses during a $60-5$ sprint performance. After an incremental test and a familiarization visit, subjects were randomly submitted in visits three and four to a performance protocol preceded by intermittent bilateral cuff inflation [ $4 \times(5 \mathrm{~min}$ of blood flow restriction +5 -min reperfusion) $]$ at either $220 \mathrm{~mm} \mathrm{Hg}$ (IPC) or $20 \mathrm{~mm} \mathrm{Hg}$ (control). To increase data reliability, each intervention was replicated, which was also in a random manner. In addition to the mean power output, the pulmonary $\dot{\mathrm{V}}_{2}$, blood lactate kinetics, and quadriceps electromyograms (EMG) were analyzed during performance and throughout 45-min of passive recovery. Results: After IPC, performance was improved by $2.1 \%$ compared to control $195 \%$ confidence intervals of 0.8 to $3.3 \%, P=0.001$ ), followed by increases in (i) the accumulated oxygen deficit, (ii) the amplitude of blood lactate kinetics, (iii) the total amount of oxygen consumed during recovery, and (iv) the overall EMG amplitude $(P<0.05)$. In addition, the ratio between EMG and power output was higher during the final third of performance after IPC $(P<0.05)$. Conclusions: These results suggest an increased skeletal muscle activation and a higher anaerobic contribution as the ultimate responses of IPC on short-term exercise performance.

Key Words: accumulated oxygen deficit, anaerobic exercise, blood lactate kinetics, EPOC, ergogenic aid, ischemic preconditioning, mean power output, sprint cycling, surface EMG, $\dot{\mathrm{V}} \mathrm{O}_{2}$ responses 


\section{INTRODUCTION}

Initially developed with the aim of protecting cardiac muscle fibres against ischemiareperfusion injury (Murry et al. 1986), ischemic preconditioning (IPC) consists of a potent endogenous mechanism that has been shown to improve human performance in the most varied exercise modalities (de Groot et al. 2010; Jean-St-Michel et al. 2011; Bailey et al. 2012; Kjeld et al. 2014; Barbosa et al. 2015). However, while the benefits of IPC on endurance performance are being consolidated (de Groot et al. 2010; Crisafulli et al. 2011; Bailey et al. 2012; Barbosa et al. 2015; Cruz et al. 2015; Kido et al. 2015), its effects on performances more reliant on the anaerobic energy metabolism are currently seen as uncertain according to a recent meta-analysis (Salvador et al. 2016).

Research has identified a greater ability for aerobic energy transduction after IPC (de Groot et al. 2010; Barbosa et al. 2015; Cruz et al. 2015; Kido et al. 2015), which has been suggested to also play a part during anaerobic events containing a substantive contribution from the aerobic pathways (Jean-St-Michel et al. 2011). However, this hypothesis has never been tested. Meanwhile, IPC has not accelerated phase II of pulmonary $\dot{\mathrm{VO}}_{2}$ onset-kinetics during severe-intensity cycling (Cruz et al. 2015; Kido et al. 2015) and improved peak and mean power output (MPO) only during the earlier stages of a repeated sprint cycling performance (Patterson et al. 2015). It is thus unclear whether increases in oxidative contribution resulting from IPC could improve performance during a predominantly anaerobic exercise. On the other hand, the improvement in endurance cycling performance after IPC was recently associated to a progressive increase in the myoelectrical activity of the vastus lateralis muscle throughout exercise (Cruz et al. 2015), which was primarily attributed to 
recruitment of additional higher-order motor units via enhanced central motor efferent command. An improved skeletal muscle activation could be particularly relevant in the context of sprint cycling exercises, as there is relatively little time for active muscles to develop force within each pedaling cycle. In a pertinent study (Barbosa et al. 2015), IPC improved the contractile rate of force development during a constant load rhythmic handgrip exercise. However, whether a higher muscular activity arising from IPC could increase the overall crank torque production and thus MPO during a self-paced cycling performance of short-duration has not yet been investigated.

We have thus examined the hypothesis that IPC could improve the MPO during a maximal cycling exercise lasting $60-\mathrm{s}$, the duration being susceptible to influences from both aerobic and anaerobic energy metabolisms (Wittekind and Beneke 2011). Additionally, we investigated the hypotheses that a higher muscle activation and/or changes in energy metabolism could be related to the ergogenic effects of IPC on anaerobic performance. Therefore, the primary aim of this study was to evaluate the effect of acute IPC of the lower limbs on anaerobic cycling performance. Our secondary aim was to investigate the IPC effects on electromyographic and metabolic responses.

\section{MATERIALS AND METHODS}

Ethical approval. This study was carried out in accordance with the guidelines contained in the Declaration of Helsinki and was approved by the Ethics Committee in Human Research of the Santa Catarina State University. The subjects were fully informed of any risks and discomforts associated with the experiments before giving their written informed consent to participate. 
Subjects. A group of 15 recreationally trained male cyclists (De Pauw et al. 2013) ranging in age from 20 to 36 years, with an average height of $176 \mathrm{~cm}$ (range: 169-188 cm) and an average body mass of $78 \mathrm{~kg}$ (range: $68-96 \mathrm{~kg}$ ), volunteered for this study. None of the subjects were receiving any pharmacological or specific dietetic treatment. All participants attended properly fed and hydrated and were instructed not to perform strenuous exercise and to abstain from alcohol on the day before each session. They were also asked to maintain the same dietary pattern throughout the experiment and to refrain from consuming caffeinated beverages for at least $2 \mathrm{~h}$ before each trial (Goldstein et al. 2010). There were no withdrawals from the study.

Study design. Subjects were required to report to the laboratory on six occasions over a 15-day period, and all tests were interspersed with approximately $48 \mathrm{~h}$ of recovery. After an incremental test and a visit designed for familiarization with the 60-s sprint cycling performance (sessions 1 and 2), subjects were randomly submitted in sessions 3 and 4 to a performance protocol preceded by either intermittent bilateral cuff inflation to $220 \mathrm{~mm} \mathrm{Hg}$ (IPC) or to $20 \mathrm{~mm} \mathrm{Hg}$ (control). To increase data reliability, the performance protocols were replicated in visits 5 and 6 , also in a random manner. The inclusion of an additional round of exercise in each condition reduces the typical error by a factor of 0.7 (i.e. $1 / \mathrm{Vn}$, where $n$ is the number of transitions), and thus improved the signal-to-noise ratio by a factor of 1.4 (Hopkins 2000). Each subject was always tested at the same time of day to minimize the effects of diurnal variation $( \pm 2 \mathrm{~h})$ in a temperature-controlled laboratory $\left(21 \pm 1^{\circ} \mathrm{C}\right)$. All cycle tests were performed on an electrodynamically braked cycle ergometer (Lode Excalibur Sport, Groningen, The Netherlands). The ergometer seat and handlebar were adjusted for 
comfort, and the settings were replicated for subsequent visits. During all tests, subjects were verbally encouraged to give their best effort and mechanical power output was measured at a sampling rate of $5 \mathrm{~Hz}$. For the assessment of the metabolic parameters, breath-by-breath $\mathrm{VO}_{2}$ was continuously registered during the performance trial and for a 45-min seated recovery and capillary blood samples were taken at specific time points (before the test and throughout the recovery period). The muscle activation responses during the 60-s sprint performances were determined via the surface electromyographic (EMG) amplitude of the vastus lateralis muscle.

Incremental exercise test and familiarization visit. In the first visit, subjects underwent an incremental test to determine the relationship between $\dot{\mathrm{V}}_{2}$ and power output, maximal oxygen uptake, and peak aerobic power. The test consisted of 3-min of unloaded baseline pedalling followed by an increase in power output of $0.5 \mathrm{~W}$ per $\mathrm{kg}$ of body mass by every three minutes. Subjects were instructed to maintain their preferred cadence for as long as possible until volitional exhaustion. In the following session, subjects carried out a $60-\mathrm{s}$ performance test for familiarization purposes. No measures were taken during this test.

Ischemic preconditioning. IPC was performed in the supine position. The occlusion cuffs were placed proximally around the upper thighs and inflated to $220 \mathrm{~mm} \mathrm{Hg}$ to restrict arterial inflow for five min. This procedure was repeated four times, each separated by five min of reperfusion, as this protocol has been successfully applied in previous studies investigating the ergogenic effects of IPC (e.g., Jean-St-Michel et al. 2011; Kjeld et al. 2014; Cruz et al. 2015; Patterson et al. 2015). Limb IPC comprising 3 to 5 cycles of 5-min inflations was found to be safe and well tolerated in both patients and healthy volunteers (Koch et al. 
2011; Gonzalez et al. 2014; Li et al. 2015). Of interest, no objective signs of neurovascular injury were observed with no subjects experiencing skin breakdown, prolonged discoloration, or temperature or pulse disparities in the treated limb. There were no cases of deep vein thrombosis or injury to the preconditioned limb, and no protocol was prematurely terminated due to subject discomfort. During the control condition, participants followed an identical protocol, but instead, the cuff was inflated only to $20 \mathrm{~mm}$ Hg (Bailey et al. 2012; Paixão et al. 2014; Cruz et al. 2015). The time between the end of the procedure and the start of the performance test was $33 \mathrm{~min}$ (Salvador et al. 2016).

60-s sprint cycling performance. Before the sprint tests, subjects were submitted to a standard 12-min warm-up protocol (set at $90 \%$ of the individual's first lactate threshold) and then were instructed to rest passively sitting on the bike. Five minutes after the warm-up, subjects performed a self-paced 60-s seated sprint cycle test. The resistance applied on the pedals was that corresponding to $7.5 \%$ of the individual body weight (Wittekind and Beneke 2011). The participants commenced tests from a stationary start after a 10-s countdown with the crank for their preferred leg positioned at $45^{\circ}$ angle to the horizontal. During the sprint, they were informed of the time elapsed every $10 \mathrm{~s}$, but were unable to see the display of the ergometer and were not informed of their performance at any stage until the end of the experimental protocol. The performance measure of interest was the MPO. Right after the end of the test, subjects slipped into a chair positioned just behind them and at the level of the seat of the cycle ergometer, where they remained seated for a period of $45 \mathrm{~min}$.

$\dot{\mathrm{VO}}_{2}$ responses. Throughout each testing protocol, cyclists wore a facemask, and respiratory gas exchange was measured breath-by-breath using an automated open-circuit 
gas analysis system (Quark CPET, Cosmed Srl, Rome, Italy). Gas analysers were always previously calibrated using ambient air and gases containing $16 \%$ oxygen and $5 \%$ carbon dioxide. The turbine flow meter used for the determination of minute ventilation was calibrated with a 3-L calibration syringe. During the incremental test, the pulmonary gas exchange was measured with the aid of a 7-L mixing chamber. The breath-by-breath gas exchange data from each test were initially examined to exclude occasional errant breaths caused by coughing, swallowing, sighing, etc., which were considered not to be reflective of the underlying kinetics; i.e. values greater than three standard deviations from the local mean were omitted. During the incremental test, individual relationships between power output and $\dot{\mathrm{VO}}_{2}$ were established using the mean $\dot{\mathrm{VO}}_{2}$ measured over the last $30 \mathrm{~s}$ of each stage for the calculation of the accumulated oxygen deficit (AOD) according to Noordhof et al. (2010), under the assumption that IPC does not affect the relationship between power output and submaximal $\mathrm{V}_{2}$ (de Groot et al. 2010; Clevidence et al. 2012). The maximal pulmonary oxygen uptake was taken as the highest 15-breaths rolling average value attained during the test. The $\mathrm{VO}_{2}$ for each 60-s cycle performance and during recovery were integrated over time to provide the accumulated gross $\mathrm{VO}_{2}$ during the sprint and the excess post-exercise oxygen consumption (EPOC), respectively. Pretest $\dot{\mathrm{VO}}_{2}$ was calculated as the average of $30 \mathrm{~s}$ preceding the sprint and peak $\mathrm{VO}_{2}$ was the highest 5 -s value attained during the test. In summary, the relevant variables derived from $\dot{\mathrm{VO}}_{2}$ measurements were: pretest $\dot{\mathrm{VO}}_{2}$, peak $\mathrm{VO}_{2}$, accumulated $\mathrm{VO}_{2}, \mathrm{AOD}$, and EPOC (gross).

Blood lactate kinetics. For the performance trials, capillary blood samples $(25 \mu \mathrm{L})$ were taken from the hyperemic earlobe (Finalgon ${ }^{\circledR}$, Thomae, Bieberach, Germany) and analysed for blood lactate concentration ([La]). Samples were collected at rest, immediately before 
the sprint test ([La] $]_{\text {pre }}$ ) and then for $45 \mathrm{~min}$ of passive recovery (every min from 0 to $10 \mathrm{~min}$, $2 \mathrm{~min}$ from 10 to $20 \mathrm{~min}$, and $5 \mathrm{~min}$ from 25 to $45 \mathrm{~min}$ ) in order to determine blood lactate kinetics. All blood samples were stored in Eppendorf ${ }^{\mathrm{TM}}$ tubes containing $50 \mu \mathrm{L}$ of $1 \% \mathrm{NaF}$ in a $-20^{\circ} \mathrm{C}$ environment. Later, samples were analysed by enzyme electrode technology (YSI 1500 SPORT, Yellow Springs, Ohio, USA). Calibration was made using a solution of $5 \mathrm{mmol} \cdot \mathrm{L}^{-1}$ (YSI 2327 Standard, Yellow Springs, Ohio, USA). The blood lactate kinetics for the performance tests was individually analysed by using the three-parameter biexponential model proposed by Beneke et al. (2010). A three-parameter model was chosen over the traditional fourparameter model because it provided more realistic parameter estimations with respect to the elimination of lactate from the blood compartment at relatively short periods of blood sampling (Beneke et al. 2007). The non-linear regression encompassed only post-exercise [La], as in the traditional four-parameter model (Freund and Gendry 1978). Therefore, this bicompartmental model required samples of [La] at rest ([La $\left.]_{\text {rest }}\right)$, immediately after exercise ([La $\left.]_{0}\right)$, and for at least $45 \mathrm{~min}$ post-exercise. It approximates the vascular increase in lactate concentration after the end of exercise $(A)$. It furthermore estimates two velocity constants describing the corresponding kinetics of the appearance $\left(K_{1}\right)$ and the disappearance $\left(K_{2}\right)$ of lactate into and out of the blood compartment. The absolute amplitude of blood lactate kinetics was calculated by summing the net increase in [La] during exercise (i.e., [La $]_{0}$ $\left.[L a]_{\text {pre }}\right)$ to the exponential term $A$. Therefore, the [La] variables of concern were: [La] $]_{\text {rest }}$ $[\mathrm{La}]_{\text {pre, }}[\mathrm{La}]_{0},[\mathrm{La}]_{\text {peak }}, A, K_{1}, K_{2}$, and absolute $A$. For more detailed informations about the bicompartimental model of blood lactate kinetics, the reader is referred to previous papers (Freund and Gendry 1978; 1981b; 1981a).

$$
[\mathrm{La}]_{(t)}=\frac{A K_{1}}{K_{2}-K_{1}}\left(e^{-K_{1} t}-e^{-K_{2} t}\right)+\left([\mathrm{La}]_{0}-[\text { La }]_{\text {rest }}\right) e^{-K_{2} t}+[\mathrm{La}]_{\text {rest }}
$$


EMG. Quadriceps EMG signals were recorded from the right vastus lateralis during the sprint using bipolar $10 \mathrm{~mm}$ diameter $\mathrm{Ag}-\mathrm{AgCl}$ surface electrodes with full-surface solid adhesive hydrogel (Tyco Healthcare Group LP, Massachusetts, USA). Prior to placing the electrodes, the skin over the muscle was shaved, sandpapered and cleaned with ethanol. The placement and location of the electrodes are according to the standard recommendations (Hermens et al. 2000). Therefore, two electrodes were carefully taped to the muscle belly, in the approximate direction of the muscle fibres with an inter-electrode distance of $20 \mathrm{~mm}$. The reference electrode was placed over an electrically neutral site (processus spinosus of $\mathrm{C7}$ vertebra). To minimize movement artefacts, electrode cables were fastened to the subject's quadriceps using adhesive tape. The positioning of the electrodes was marked with indelible ink to ensure that they were placed in the same location at subsequent visits. EMG was recorded with a common mode rejection ratio of $110 \mathrm{~dB}$ and an input impedance of $10 \mathrm{G} \Omega$ with a four-channel EMG system (Miotool 400 USB, Miotec Ltda., Porto Alegre, Brazil). The EMG signals were amplified ( $\times 400), A / D$ converted (14 bits resolution) at a sampling rate of $2 \mathrm{kHz}$, and stored for later analysis. The raw digital EMG signal was processed using MATLAB 7.12 (MathWorks Inc., Natick, EUA). The signal was initially filtered using a $10-500 \mathrm{~Hz}$ band pass, fifth-order Butterworth filter. The data were then rectified and smoothed using root mean squared analysis (RMS), calculated for a $25 \mathrm{~ms}$ window. A computer algorithm identified the onset/offset of activity where the signal deviated by more than four standard deviations above the baselines. Each EMG burst was inspected to verify the timing identified by the computer. The average RMS over the periods of muscle activity (5-s intervals) were then calculated and constituted the myoelectric variable of interest. 
Statistical analysis. Comparisons were performed with the mixed linear modelling procedure of the IBM SPSS statistics (Version 19.0, IBM Corporation, New York, USA), followed by specific pairwise comparisons (Fisher's least significant difference tests) when appropriate. The Subject term was inserted as a random effect. Condition (i.e. control vs. IPC) and Time (where applicable) were the fixed effects. However, the Order in which the interventions were applied was also included as an additional fixed effect to account for continuing familiarization or other order effects. Prior to the mixed modeling, data were logtransformed by taking natural logarithms of the raw measures and subsequently multiplied by 100 . This procedure was performed to reduce non-uniformity of effects and errors (Hopkins 2000; Hopkins et al. 2009). In addition, this approach yields effects and variabilities as percentages of the mean, which are the natural metric for most measures of athletic performance (Hopkins 2000). After analysis, the mean effect estimates, the typical errors of measurement, and its corresponding 95\% confidence intervals (all in log units $\times 100$ ) were converted into exact percentages: $100\left(e^{x} / 100-1\right)$, where $x$ is the change in the mean, the typical error, or the lower/upper confidence bound. Similarly, the estimated marginal means of interest and its corresponding standard error (SE) intervals were back-transformed into raw units: $\mathrm{e}^{(\mathrm{x} / 100)}$. As the errors become slightly asymmetrical after back transformation, graphs were presented using a log scale on the y-axis (Hopkins et al. 2009). Typical errors of the measurement were derived by back transformation of the square root of the Residual term after any interaction between Subject and Condition (i.e. individual responses to the IPC procedure) has been taken into account (Hopkins 2000). The uncertainties in the effects and errors were always expressed as $95 \%$ confidence intervals $(\mathrm{Cl})$ and all tests were analysed at an alpha level of 0.05 . 


\section{RESULTS}

Incremental exercise test. Subjects attained a peak aerobic power of 297 [289-306] W, while the maximal oxygen uptake was $51[50-53] \mathrm{mL} \cdot \mathrm{kg}^{-1} \cdot \mathrm{min}^{-1}$.

60-s sprint cycling performance. The typical variation of the subject's MPO from test to test was $2.2 \%(95 \% \mathrm{Cl}$ of 1.8 to $2.8 \%)$. After the application of IPC, MPO was improved by $2.1 \%$ relative to control $(95 \% \mathrm{Cl}$ of 0.8 to $3.3 \%, \mathrm{P}=0.001)$. The marginal means and $\mathrm{SE}$ intervals for control and IPC conditions were 528 [517-540] and 539 [528-551] W, respectively. When MPO was expressed in 5-s intervals, the performance improvement was higher at the exercise onset $(6.8 \%, 95 \% \mathrm{Cl}$ of 1.2 to $12.8 \%, \mathrm{P}=0.017)$, but then decreased progressively throughout the sprint (Figure 1).

Metabolic responses. The comparisons of the metabolic responses are presented in Table 1. There were no significant differences in any of the $\mathrm{VO}_{2}$ responses during exercise, with the accumulated $\mathrm{VO}_{2}$ being similar between conditions. Conversely, the AOD was significantly higher after IPC than after control. Furthermore, during the $45 \mathrm{~min}$ of seated recovery, subjects presented a larger EPOC in the IPC condition. Figure 2 illustrates the blood lactate concentration reponses during recovery in each condition together with the respective fitted responses. The $[\mathrm{La}]_{\text {pre, }} A$ and the absolute $A$ of the blood lactate kinetics were significantly higher after IPC compared to control (Table 1).

EMG. The temporal profiles of the EMG signal throughout the sprint in each condition are illustrated in Figure 3A. The typical error of the non-normalized EMG measurements was $13.7 \%(95 \% \mathrm{Cl}$ of 12.8 to $14.6 \%)$, and the overall EMG activity of the vastus lateralis was $9.7 \%$ 
higher after IPC $(95 \% \mathrm{Cl}$ of 2.0 to $18.0 \%, \mathrm{P}=0.017)$. Pairwise comparisons revealed significant changes toward an increased response in the $15-20$ and $40-55 \mathrm{~s}$ time intervals compared with control $(P \leq 0.030)$. Figure $3 B$ illustrates the influence of IPC on the ratio between EMG activity and absolute power output (EMG/PO ratio). There was a marginal trend for the overall EMG/PO ratio to be higher after IPC $(P=0.065)$ and pairwise comparisons indicated significant increases after IPC during the 40-55 s time intervals when compared to control $(\mathrm{P} \leq 0.042)$.

\section{DISCUSSION}

The major original findings of this investigation include the following. (i) Relative to control, preconditioning of the lower limbs improved MPO by $2.1 \%$ during a short-term cycling performance in recreational cyclists. This result is in agreement with the earlier observations of Jean-St-Michel et al. (2011), who were the first to demonstrate the benefits of IPC on anaerobic performance of similar duration in highly trained swimmers. (ii) The performance change was accompanied by modifications in anaerobic metabolism, rather than aerobic, and in the electromyographic responses, suggesting an increase in skeletal muscle activation and a higher anaerobic contribution as the ultimate responses caused by IPC on short-term exercise performance.

Studies investigating the effect of IPC on anaerobic cycling are accompanied by mixed results in terms of performance. For example, Paixão et al. (2014) found a detrimental acute effect of IPC during a series of Wingate tests in amateur cyclists. On the other hand, in the study of Patterson et al. (2015), IPC improved peak and mean power output during the first three sprints of a repeated sprint cycling test in active subjects. While we cannot fully 
explain the discrepancies between our findings and the lack of benefit reported in earlier investigations, it was noticed that the success of the IPC intervention on anaerobic events might be dependent on the time between the IPC intervention and the chosen performance test (Patterson et al. 2015). Evidence supporting this hypothesis was provided in a recent meta-analysis (Salvador et al. 2016). If so, this helps explain why exercise performances reliant on anaerobic processes are not improved with IPC when shorter times of 5-16 min are employed (Crisafulli et al. 2011; Gibson et al. 2013; Paixão et al. 2014; Gibson et al. 2015; Lalonde and Curnier 2015) compared to longer periods (present study, Jean-St-Michel et al. 2011; Patterson et al. 2015). Other factors may also contribute for the heterogeneity in the effect between studies, as participant characteristics (e.g., sex, training status) and study methods (e.g., exercise mode, pre-study restrictions, IPC protocol) differ widely (Incognito et al. 2015). Nevertheless, since the magnitude of performance improvement was similar to the typical error of the measurement, the present results indicate that IPC can evoke moderate enhancements in long sprint cycling performance for recreational cyclists (please see Hopkins et al. 1999), at least when IPC is performed around 30 min before performance. Furthermore, as the benefit was greater at the early stages of exercise, these results are promising for future studies investigating athletes competing in track cycling events of similar duration, like the $1-\mathrm{km}$ time trial, as a fast first $250-\mathrm{m}$ split time is paramount for quick performance times (Corbett 2009).

The higher $A O D$ and amplitude of blood lactate kinetics under unaltered $\mathrm{VO}_{2}$ responses indicates for the first time an increased anaerobic contribution after IPC. This scenario is additionally supported by the higher EPOC, in that much of the post-exercise $\mathrm{VO}_{2}$ reflects the replenishment of the high-energy phosphate compounds and the removal of the lactate 
generated during exercise (Gaesser and Brooks 1984; Wittekind and Beneke 2011). However, it should be noted that neither of the two previous studies in which anaerobic performance was enhanced with IPC witnessed significant changes in post-exercise blood lactate concentrations (Jean-St-Michel et al. 2011; Patterson et al. 2015). In our opinion, a possible explanation may include the use of [La] measurements at fixed time points to assess the glycolytic contribution, which does not allow for alterations induced by IPC on [La] kinetics. Such cases should be considered with care because the [La] does reflect not only an increase in lactate in the working muscle space but also a number of other processes modulating the transport of lactate into and its elimination out of the blood compartment. The bicompartmental model is sensitive to variations in the lactate exchange and removal abilities, thus providing a better index of whole-body lactate accumulation. Although further studies are necessary to investigate the etiology of the higher muscle activation potentials following IPC, an overshoot in efferent neural drive from spinal $\alpha$ motoneuron pool has been suggested to play a pivotal function by recruiting additional motor units, thereby allowing the use of a higher fraction of the locomotor muscle 'functional reserve' (Crisafulli et al. 2011; Cruz et al. 2015). Therefore, together with the increased EMG/PO ratio mostly during the final third of performance, these findings could be suggesting a more severe degree of skeletal muscle fatigue at the end of exercise in the IPC condition (when relative to pre-exercise levels), defined as a reduction in muscle force or power output for a given muscle activation (Grassi et al. 2015). If so, IPC could be possibly allowing subjects to exercise slightly beyond their individual critical threshold of fatigue for the task (Amann et al. 2009; Crisafulli et al. 2011), which requires investigation. Meanwhile, in the study of Patterson et al. (2015), the rate of change in the median frequency of the 
power spectral density of the EMG signal (associated with localized muscular fatigue) was possibly higher in the IPC trial.

In the present study, the IPC-induced benefit on MPO was markedly higher at exercise onset, but decreased throughout performance, with virtually no changes during the last few seconds of exercise. As the participants were repeatedly asked to reproduce the pattern of work rate distribution in all performance visits, it is possible that such changes originates from a less conservative pacing strategy adopted by the higher brain centres (Hampson et al. 2001). Indeed, IPC was recently shown to reduce the perception of effort during severeintensity cycling (Cruz et al. 2015). On the other hand, decreased inhibition or changes in the intrinsic properties of $\alpha$-motoneurons could also have a role at the spinal level. Importantly, both of these outcomes are expected to increase the voluntary neural drive/output to the active muscles, which is in agreement with our observations of higher EMG activity reported herein for sprint and elsewhere for endurance performance (Cruz et al. 2015). However, while the effects of IPC on the supraspinal sites of motor control during exercise remains to be investigated, the higher EMG activity and lower decline in isometric force after IPC observed in feline hindlimbs during prolonged ischemia (submitted to electrical stimulation of the sciatic nerve) could be suggesting a lesser degree of central fatigue already at the spinal level (Phillips et al. 1997). In practical terms, a higher neural drive could be increasing the rate of force development during exercise, which would be very important in the context of sprint exercises. Although we were not able to measure the rate of force development in the present study, it was recently shown to be improved by IPC during a handgrip exercise (Barbosa et al. 2015). 
Currently, little is known about the mechanisms underlying the ergogenic effects of IPC. It has been suggested that IPC lowers the sensitivity of the body to fatiguing signals (Crisafulli et al. 2011; Cruz et al. 2015; Salvador et al. 2016). Indeed, activation of opioid receptors in the striated muscle tissue by circulating endogenous opioid peptides has been associated with the early phase of protection of either local acute or remote IPC (Addison et al. 2003; Dragasis et al. 2013). Interestingly, these receptors are also present in terminal endings of group III and IV muscle afferents (Leal et al. 2013), which are known to modulate exercise performance by facilitating central fatigue (Amann et al. 2011). Therefore, albeit speculative, it is possible that activation of opioid receptors could have decreased the excitability of peripheral nerve terminals after the IPC procedure, resulting in lower spontaneous discharges to the central nervous system (Tsuchimochi et al. 2010; Amann 2012). Interestingly, blockade of III/IV muscle afferents with fentanyl has been shown to increase central motor drive and thus peripheral fatigue during high-intensity cycling, as well as to influence pacing and the ratings of perceived exertion (Amann et al. 2009; Amann et al. 2011). These responses are similar to those observed in the current study and by Cruz et al. (2015), suggesting that the opioid-mediated muscle afferents may indeed play a role in the effects of IPC on exercise performance. However, a more targeted approach should be conducted, maybe investigating the interaction between IPC and nonselective opioid receptor antagonists.

Perhaps the main methodological constraint of all IPC studies is the inability to completely "blind" the subjects, because the sensations induced by the IPC and the low-pressure protocol are clearly distinct. We are unaware of any method that could satisfactorily circumvent this limitation while, at the same time, allowing for a proper investigation of the 
particular hypotheses of the present study. To reduce potential bias, subjects were all deprived from any information until the end of the data collection, and they were always verbally encouraged to realize their maximum effort. However, a placebo (and nocebo) effect cannot yet be discarded (Marocolo et al. 2016). Secondly, as we employed a time window of approximately $48 \mathrm{~h}$ between performance visits, the extent at which the less intense second window of protection lasting $48-72 \mathrm{~h}$ could have affected the results is unknown (Marber et al. 1993). In an attempt to mitigate this limitation, the order of trials was included as an additional fixed effect in the mixed model. However, if a "residual" ergogenic effect of IPC also remains for few days, this limited washout period may have eventually enlarged the typical error of some important measures. Finally, one could argue that the use of non-normalized EMG is unreliable. Although the lack of baseline EMG measurements is indeed a limitation, as its use could markedly improve reliability, our design involved two rounds of exercise in each condition, which should have reduced the typical error from 13.7 to approximately 9.7\% (Hopkins 2000). This value proved sensitive to detect the overall increase in skeletal muscle activation of $9.7 \%$ after IPC. Furthermore, it made possible to discriminate superior changes (10.4-14.3\%) at specific time points.

In conclusion, this study has demonstrated that acute IPC of the lower limbs improves the 60-s sprint performance of recreational cyclists. While a higher skeletal muscle activation seems to be a common response of IPC irrespective of exercise duration, the ergogenic benefit of IPC on short-duration exercise performance could not be linked to an enhanced aerobic energy metabolism, but rather to a greater anaerobic contribution.

\section{ACKNOWLEDGMENTS}


Thanks to all colleagues who contributed and to CNPq and FAPESC for their financial support.

\section{CONFLICT OF INTEREST}

The authors declare that have no conflict of interest. The results of the current study do not constitute endorsement of the product by the authors or the journal. 


\section{REFERENCES}

Addison, P.D., Neligan, P.C., Ashrafpour, H., Khan, A., Zhong, A., Moses, M., Forrest, C.R., and Pang, C.Y. 2003. Noninvasive remote ischemic preconditioning for global protection of skeletal muscle against infarction. Am. J. Physiol. Heart Circ. Physiol. 285(4): H1435-1443. doi: 10.1152/ajpheart.00106.2003.

Amann, M. 2012. Significance of Group III and IV muscle afferents for the endurance exercising human. Clin. Exp. Pharmacol. Physiol. 39(9): 831-835. doi: 10.1111/j.14401681.2012.05681.x.

Amann, M., Blain, G.M., Proctor, L.T., Sebranek, J.J., Pegelow, D.F., and Dempsey, J.A. 2011. Implications of group III and IV muscle afferents for high-intensity endurance exercise performance in humans. J. Physiol. 589(Pt 21): 5299-5309. doi: 10.1113/jphysiol.2011.213769.

Amann, M., Proctor, L.T., Sebranek, J.J., Pegelow, D.F., and Dempsey, J.A. 2009. Opioidmediated muscle afferents inhibit central motor drive and limit peripheral muscle fatigue development in humans. J. Physiol. 587(Pt 1): 271-283. doi: 10.1113/jphysiol.2008.163303.

Bailey, T.G., Jones, H., Gregson, W., Atkinson, G., Cable, N.T., and Thijssen, D.H. 2012. Effect of ischemic preconditioning on lactate accumulation and running performance. Med. Sci. Sports Exerc. 44(11): 2084-2089. doi: 10.1249/MSS.0b013e318262cb17.

Barbosa, T.C., Machado, A.C., Braz, I.D., Fernandes, I.A., Vianna, L.C., Nobrega, A.C., and Silva, B.M. 2015. Remote ischemic preconditioning delays fatigue development during handgrip exercise. Scand. J. Med. Sci. Sports 25(3): 356-364. doi: 10.1111/sms.12229.

Beneke, R., Jumah, M.D., and Leithauser, R.M. 2007. Modelling the lactate response to short-term all out exercise. Dyn. Med. 6(10). doi: 10.1186/1476-5918-6-10.

Beneke, R., Wittekind, A., Muhling, M., Bleif, I., and Leithauser, R.M. 2010. Lactate response to short term exercise with elevated starting levels. Eur. J. Appl. Physiol. 110(1): 215-218. doi: 10.1007/s00421-010-1481-z.

Clevidence, M.W., Mowery, R.E., and Kushnick, M.R. 2012. The effects of ischemic preconditioning on aerobic and anaerobic variables associated with submaximal cycling performance. Eur. J. Appl. Physiol. 112(10): 3649-3654. doi: 10.1007/s00421-012-2345-5. 
Corbett, J. 2009. An analysis of the pacing strategies adopted by elite athletes during track cycling. Int. J. Sports Physiol. Perform. 4(2): 195-205. Available from http://www.ncbi.nlm.nih.gov/pubmed/19567923.

Crisafulli, A., Tangianu, F., Tocco, F., Concu, A., Mameli, O., Mulliri, G., and Caria, M.A. 2011. Ischemic preconditioning of the muscle improves maximal exercise performance but not maximal oxygen uptake in humans. J. Appl. Physiol. (1985) 111(2): 530-536. doi: 10.1152/japplphysiol.00266.2011.

Cruz, R.S., de Aguiar, R.A., Turnes, T., Pereira, K.L., and Caputo, F. 2015. Effects of ischemic preconditioning on maximal constant-load cycling performance. J. Appl. Physiol. (1985) 119(9): 961-967. doi: 10.1152/japplphysiol.00498.2015.

de Groot, P.C., Thijssen, D.H., Sanchez, M., Ellenkamp, R., and Hopman, M.T. 2010. Ischemic preconditioning improves maximal performance in humans. Eur. J. Appl. Physiol. 108(1): 141-146. doi: 10.1007/s00421-009-1195-2.

De Pauw, K., Roelands, B., Cheung, S.S., de Geus, B., Rietjens, G., and Meeusen, R. 2013. Guidelines to classify subject groups in sport-science research. Int. J. Sports Physiol. Perform. 8(2): 111-122. Available from http://www.ncbi.nlm.nih.gov/pubmed/23428482.

Dragasis, S., Bassiakou, E., lacovidou, N., Papadimitriou, L., Andreas Steen, P., Gulati, A., and Xanthos, T. 2013. The role of opioid receptor agonists in ischemic preconditioning. Eur. J. Pharmacol. 720(1-3): 401-408. doi: 10.1016/j.ejphar.2013.10.001.

Freund, H. and Gendry, P. 1978. Lactate kinetics after short strenuous exercise in man. Eur. J. Appl. Physiol. Occup. Physiol. 39(2): 123-135. Available from http://www.ncbi.nlm.nih.gov/pubmed/689008.

Gaesser, G.A. and Brooks, G.A. 1984. Metabolic bases of excess post-exercise oxygen consumption: a review. Med. Sci. Sports Exerc. 16(1): 29-43. Available from http://www.ncbi.nlm.nih.gov/pubmed/6369064.

Gibson, N., Mahony, B., Tracey, C., Fawkner, S., and Murray, A. 2015. Effect of ischemic preconditioning on repeated sprint ability in team sport athletes. J. Sports Sci. 33(11): 11821188. doi: 10.1080/02640414.2014.988741.

Gibson, N., White, J., Neish, M., and Murray, A. 2013. Effect of ischemic preconditioning on land-based sprinting in team-sport athletes. Int. J. Sports Physiol. Perform. 8(6): 671-676. Available from http://www.ncbi.nlm.nih.gov/pubmed/23578975. 
Goldstein, E.R., Ziegenfuss, T., Kalman, D., Kreider, R., Campbell, B., Wilborn, C., Taylor, L., Willoughby, D., Stout, J., Graves, B.S., Wildman, R., Ivy, J.L., Spano, M., Smith, A.E., and Antonio, J. 2010. International society of sports nutrition position stand: caffeine and performance. J. Int. Soc. Sports Nutr. 7(1): 5. doi: 10.1186/1550-2783-7-5.

Gonzalez, N.R., Connolly, M., Dusick, J.R., Bhakta, H., and Vespa, P. 2014. Phase I clinical trial for the feasibility and safety of remote ischemic conditioning for aneurysmal subarachnoid hemorrhage. Neurosurgery 75(5): 590-598; discussion $598 . \quad$ doi: 10.1227/NEU.0000000000000514.

Grassi, B., Rossiter, H.B., and Zoladz, J.A. 2015. Skeletal muscle fatigue and decreased efficiency: two sides of the same coin? Exerc. Sport Sci. Rev. 43(2): 75-83. doi: 10.1249/JES.0000000000000043.

Hampson, D.B., St Clair Gibson, A., Lambert, M.I., and Noakes, T.D. 2001. The influence of sensory cues on the perception of exertion during exercise and central regulation of exercise performance. Sports Med. 31(13): 935-952. Available from http://www.ncbi.nIm.nih.gov/pubmed/11708402.

Hermens, H.J., Freriks, B., Disselhorst-Klug, C., and Rau, G. 2000. Development of recommendations for SEMG sensors and sensor placement procedures. J. Electromyogr. Kinesiol. 10(5): 361-374. Available from http://www.ncbi.nlm.nih.gov/pubmed/11018445.

Hopkins, W.G. 2000. Measures of reliability in sports medicine and science. Sports Med. 30(1): 1-15. Available from http://www.ncbi.nlm.nih.gov/pubmed/10907753.

Hopkins, W.G., Hawley, J.A., and Burke, L.M. 1999. Design and analysis of research on sport performance enhancement. Med. Sci. Sports Exerc. 31(3): 472-485. Available from http://www.ncbi.nlm.nih.gov/pubmed/10188754.

Hopkins, W.G., Marshall, S.W., Batterham, A.M., and Hanin, J. 2009. Progressive statistics for studies in sports medicine and exercise science. Med. Sci. Sports Exerc. 41(1): 3-13. doi: 10.1249/MSS.0b013e31818cb278.

Incognito, A.V., Burr, J.F., and Millar, P.J. 2015. The effects of ischemic preconditioning on human exercise performance. Sports Med. doi: 10.1007/s40279-015-0433-5. In press

Jean-St-Michel, E., Manlhiot, C., Li, J., Tropak, M., Michelsen, M.M., Schmidt, M.R., McCrindle, B.W., Wells, G.D., and Redington, A.N. 2011. Remote preconditioning improves maximal performance in highly trained athletes. Med. Sci. Sports Exerc. 43(7): 1280-1286. doi: 10.1249/MSS.0b013e318206845d. 
Kido, K., Suga, T., Tanaka, D., Honjo, T., Homma, T., Fujita, S., Hamaoka, T., and Isaka, T. 2015. Ischemic preconditioning accelerates muscle deoxygenation dynamics and enhances exercise endurance during the work-to-work test. Physiol. Rep. 3(5): e12395-e12395. doi: 10.14814 phy2.12395.

Kjeld, T., Rasmussen, M.R., Jattu, T., Nielsen, H.B., and Secher, N.H. 2014. Ischemic preconditioning of one forearm enhances static and dynamic apnea. Med. Sci. Sports Exerc. 46(1): 151-155. doi: 10.1249/MSS.0b013e3182a4090a.

Koch, S., Katsnelson, M., Dong, C., and Perez-Pinzon, M. 2011. Remote ischemic limb preconditioning after subarachnoid hemorrhage: a phase $\mathrm{Ib}$ study of safety and feasibility. Stroke 42(5): 1387-1391. doi: 10.1161/STROKEAHA.110.605840.

Lalonde, F. and Curnier, D.Y. 2015. Can anaerobic performance be improved by remote ischemic preconditioning? J. Strength Cond. Res. 29(1): 80-85. doi: 10.1519/JSC.0000000000000609.

Leal, A.K., Yamauchi, K., Kim, J., Ruiz-Velasco, V., and Kaufman, M.P. 2013. Peripheral deltaopioid receptors attenuate the exercise pressor reflex. Am. J. Physiol. Heart Circ. Physiol. 305(8): H1246-1255. doi: 10.1152/ajpheart.00116.2013.

Li, S., Ma, C., Shao, G., Esmail, F., Hua, Y., Jia, L., Qin, J., Ren, C., Luo, Y., Ding, Y., Borlongan, C.V., and Ji, X. 2015. Safety and Feasibility of Remote Limb Ischemic Preconditioning in Patients With Unilateral Middle Cerebral Artery Stenosis and Healthy Volunteers. Cell Transplant. 24(9): 1901-1911. doi: 10.3727/096368914X683520.

Marber, M.S., Latchman, D.S., Walker, J.M., and Yellon, D.M. 1993. Cardiac stress protein elevation 24 hours after brief ischemia or heat stress is associated with resistance to myocardial infarction. Circulation 88(3): 1264-1272. Available from http://www.ncbi.nlm.nih.gov/pubmed/8353888.

Marocolo, M., da Mota, G.R., Simim, M.A., and Appell Coriolano, H.J. 2016. Myths and Facts About the Effects of Ischemic Preconditioning on Performance. Int. J. Sports Med. 37(02): 87-96. doi: 10.1055/s-0035-1564253.

Murry, C.E., Jennings, R.B., and Reimer, K.A. 1986. Preconditioning with ischemia: a delay of lethal cell injury in ischemic myocardium. Circulation 74(5): 1124-1136. Available from http://www.ncbi.nlm.nih.gov/pubmed/3769170. 
Noordhof, D.A., de Koning, J.J., and Foster, C. 2010. The maximal accumulated oxygen deficit method: a valid and reliable measure of anaerobic capacity? Sports Med. 40(4): 285-302. doi: 10.2165/11530390-000000000-00000.

Paixão, R.C., da Mota, G.R., and Marocolo, M. 2014. Acute effect of ischemic preconditioning is detrimental to anaerobic performance in cyclists. Int. J. Sports Med. 35(11): 912-915. doi: 10.1055/s-0034-1372628.

Patterson, S.D., Bezodis, N.E., Glaister, M., and Pattison, J.R. 2015. The Effect of Ischemic Preconditioning on Repeated Sprint Cycling Performance. Med. Sci. Sports Exerc. 47(8): 1652-1658. doi: 10.1249/MSS.0000000000000576.

Phillips, D.J., Petrie, S.G., Zhou, B.H., Guanche, C.A., and Baratta, R.V. 1997. Myoelectric and mechanical changes elicited by ischemic preconditioning in the feline hindlimb. J. Electromyogr. $\quad$ Kinesiol. $\quad$ 7(3): 187-192. Available from http://www.ncbi.nIm.nih.gov/pubmed/20719704.

Salvador, A.F., De Aguiar, R.A., Lisboa, F.D., Pereira, K.L., Cruz, R.S., and Caputo, F. 2016. Ischemic Preconditioning and Exercise Performance: A Systematic Review and MetaAnalysis. Int. J. Sports Physiol. Perform. 11(1): 4-14. doi: 10.1123/ijspp.2015-0204.

Tsuchimochi, H., McCord, J.L., and Kaufman, M.P. 2010. Peripheral mu-opioid receptors attenuate the augmented exercise pressor reflex in rats with chronic femoral artery occlusion. Am. J. Physiol. Heart Circ. Physiol. 299(2): H557-565. doi: 10.1152/ajpheart.00387.2010.

Wittekind, A. and Beneke, R. 2011. Metabolic and performance effects of warm-up intensity on sprint cycling. Scand. J. Med. Sci. Sports 21(6): e201-207. doi: 10.1111/j.16000838.2010.01248.x.

Zouloumian, P. and Freund, H. 1981a. Lactate after exercise in Man: II. Mathematical model. Eur. J. Appl. Physiol. Occup. Physiol. 46(2): 135-147. Available from http://www.ncbi.nlm.nih.gov/pubmed/7194791.

Zouloumian, P. and Freund, H. 1981b. Lactate after exercise in man: III. Properties of the compartment model. Eur. J. Appl. Physiol. Occup. Physiol. 46(2): 149-160. Available from http://www.ncbi.nlm.nih.gov/pubmed/7194792. 
Table 1. Effects of ischemic preconditioning on metabolic responses to the 60-s sprint cycling performance.

\begin{tabular}{|c|c|c|c|c|c|c|c|c|c|}
\hline & \multicolumn{4}{|c|}{ Mean and SE interval } & \multirow{2}{*}{\multicolumn{2}{|c|}{$\begin{array}{c}\text { Typical error }(\%)^{a} \text { and } \\
95 \% \mathrm{Cl}\end{array}$}} & \multirow{2}{*}{\multicolumn{2}{|c|}{$\begin{array}{c}\text { Effect }(\%) \text { and } \\
95 \% \mathrm{Cl}\end{array}$}} & \multirow{2}{*}{ P valu } \\
\hline & & Control & & IPC & & & & & \\
\hline \multicolumn{10}{|l|}{$\dot{\mathrm{V}} \mathrm{O}_{2}$ responses } \\
\hline Pretest $\dot{\mathrm{V}}_{2}, \mathrm{~L} \cdot \mathrm{min}^{-1}$ & 0.69 & {$[0.66-0.73]$} & 0.71 & [0.67-0.75] & 15.8 & [12.6 to 19.9 ] & 2.3 & {$[-5.6$ to 10.9$]$} & 0.569 \\
\hline Accumulated $\mathrm{VO}_{2}$ (gross), L & 2.55 & {$[2.48-2.62]$} & 2.55 & {$[2.48-2.63]$} & 5.1 & [3.9 to 6.8$]$ & 0.2 & {$[-3.1$ to 3.6$]$} & 0.907 \\
\hline$A O D, L$ & 3.85 & {$[3.70-4.00]$} & 3.98 & {$[3.83-4.14]$} & 5.1 & [3.9 to 6.7$]$ & 3.4 & [0.1 to 6.8 ] & $0.045 *$ \\
\hline EPOC (gross), L & 26.83 & {$[26.21-27.46]$} & 27.45 & {$[26.82-28.10]$} & 3.8 & [3.1 to 4.8$]$ & 2.3 & [0.2 to 4.5$]$ & $0.031 *$ \\
\hline$[\text { La }]_{\text {pres }} \mathrm{mmol} \cdot \mathrm{L}^{-1}$ & 0.9 & {$[0.8-1.1]$} & 1.0 & {$[0.9-1.2]$} & 18.4 & [14.7 to 23.2$]$ & 11.6 & [1.7 to 22.4$]$ & $0.022 *$ \\
\hline$[\mathrm{La}]_{0}, \mathrm{mmol} \cdot \mathrm{L}^{-1}$ & 5.9 & {$[5.5-6.5]$} & 5.9 & {$[5.4-6.4]$} & 11.9 & [9.5 to 14.8 ] & -0.9 & {$[-6.8$ to 5.4$]$} & 0.767 \\
\hline$[\mathrm{La}]_{\text {peak }}, \mathrm{mmol} \cdot \mathrm{L}^{-1}$ & 13.1 & {$[12.6-13.6]$} & 13.6 & {$[13.1-14.2]$} & 9.9 & [7.9 to 12.4$]$ & 4.3 & {$[-1.0$ to 9.8$]$} & 0.111 \\
\hline$A, \mathrm{mmol} \cdot \mathrm{L}^{-1}$ & 10.6 & {$[9.9-11.2]$} & 11.7 & [11.0-12.4] & 11.6 & [9.3 to 14.6 ] & 10.7 & [4.2 to 17.6 ] & $0.001 *$ \\
\hline$K_{1}, \min ^{-1}$ & 0.32 & {$[0.30-0.34]$} & 0.30 & [0.28-0.32] & 28.0 & [22.1 to 35.6 ] & -6.4 & {$[-18.2$ to 7.1$]$} & 0.327 \\
\hline
\end{tabular}

Data are back-transformed means and standard errors (lower and upper bounds).

${ }^{a}$ Multiply by 0.7 to correct by the number of trials in each condition.

$* \mathrm{P} \leq 0.05$ 


\section{FIGURE LEGENDS}

Fig. 1 Mean absolute power output per 5-s interval of the 60-s sprint cycling performance for ischemic preconditioning (black bars) and control (white bars). Values are means \pm standard errors plotted on semi-log coordinates (left y-axis). The closed squares represent the percent changes induced by ischemic preconditioning relative to control (right y-axis). Note the higher benefit at the exercise onset, followed by a progressive decrease throughout.

$* P=0.017$

Fig. 2 Blood lactate responses to the 60-s sprint cycling performance under control (open circles) and IPC (closed circles) conditions, together with the mean fitted responses. As the non-linear regressions were performed individually in the raw [La] measures and only the regression parameters were log-transformed for the analysis, values are illustrated as means and standard errors of the raw data plotted on linear coordinates. Note the higher blood lactate concentrations after IPC.

Fig. 3 Myoelectrical activity of vastus lateralis (5-s intervals) during the 60-s sprint cycling performance. Figure $3 \mathrm{~A}$ represents the non-normalized EMG amplitude responses and Figure $3 \mathrm{~B}$ illustrates the ratios between electrical activity and power output (EMG/PO). Values are means \pm standard errors plotted on semi-log coordinates.

$* \mathrm{P} \leq 0.042$ 


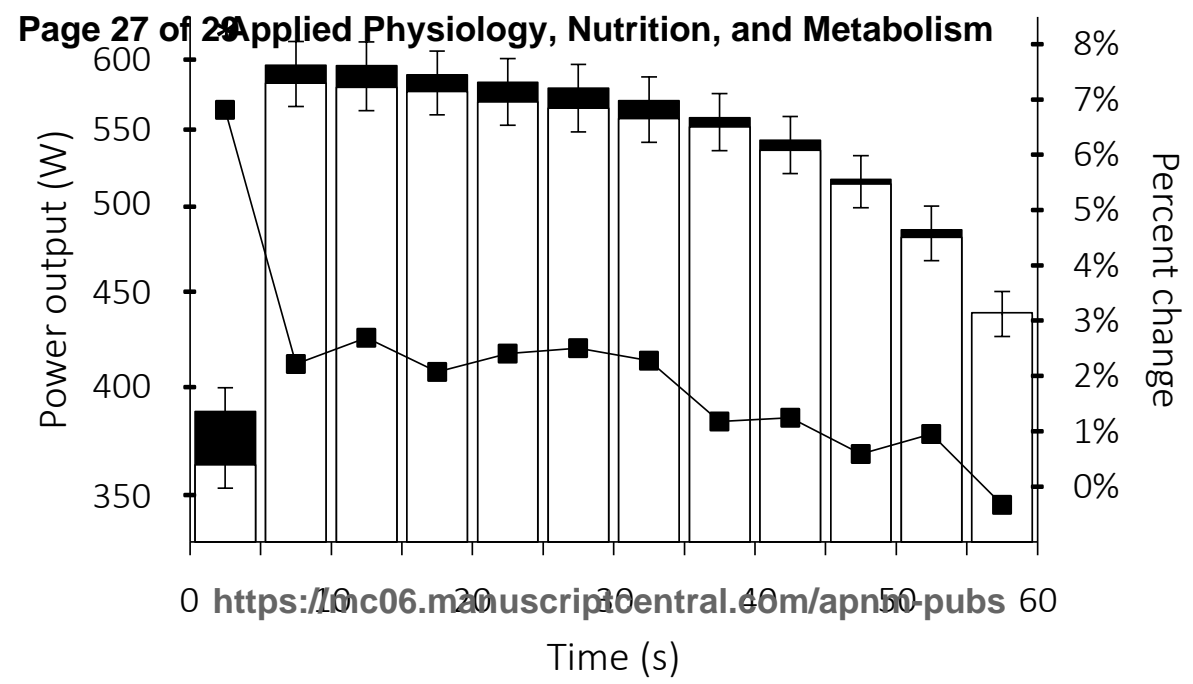




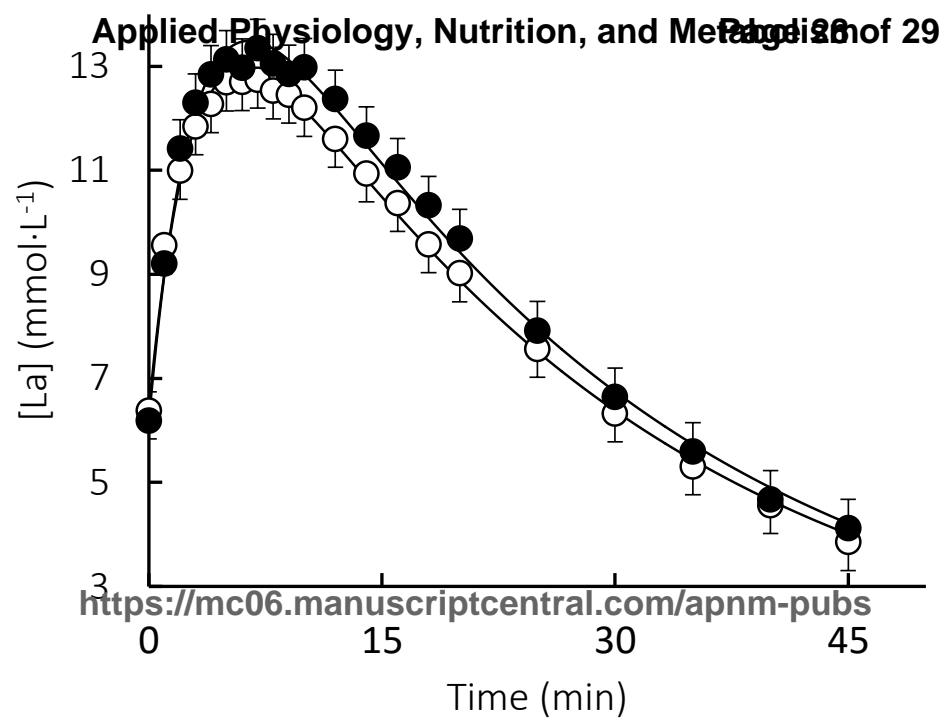


Pages39 of 29pplied Physiology, Nutrition, and Meta6̈olisw

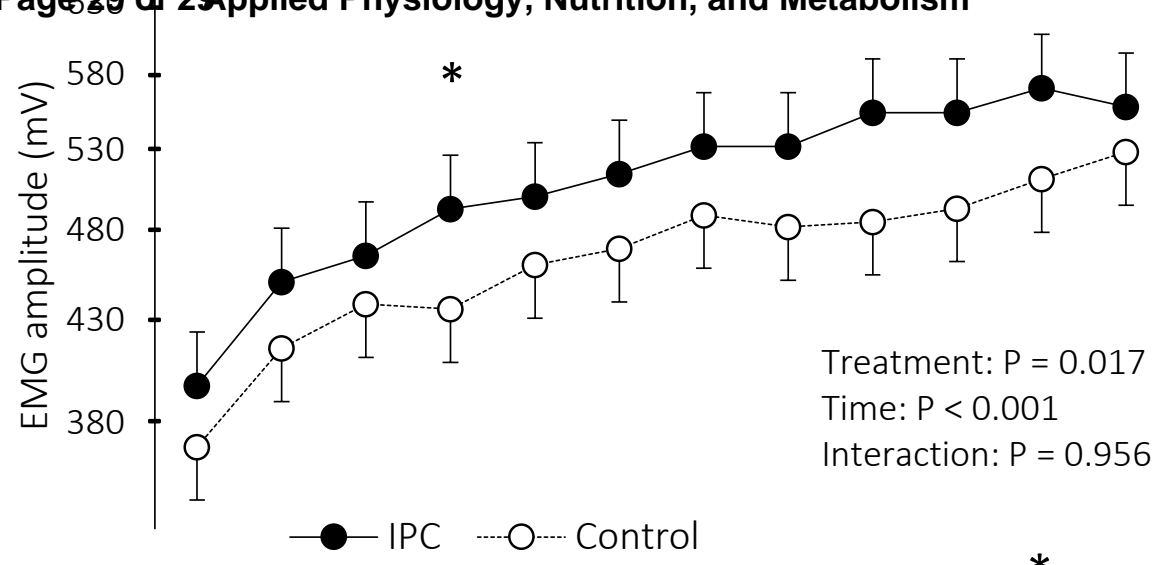

B

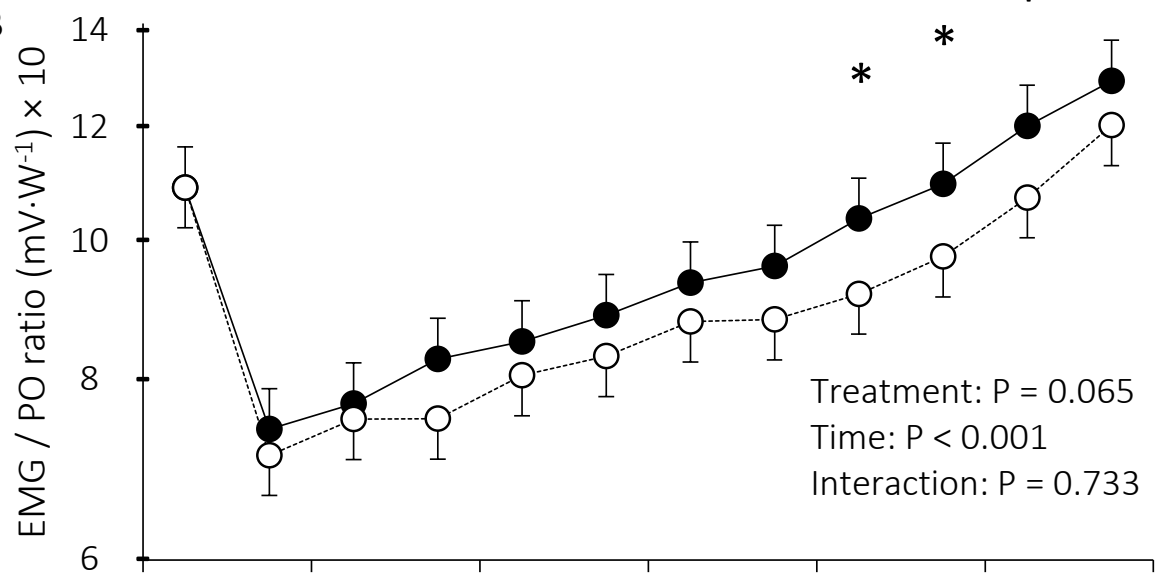

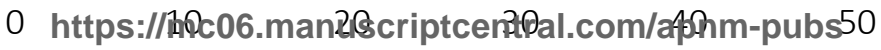

60

Time (s) 